\title{
Drugs in development for small cell lung cancer
}

\author{
Michael T. Serzan ${ }^{1}$, Saira Farid ${ }^{2}$, Stephen V. Liu ${ }^{1}$ \\ ${ }^{1}$ Lombardi Comprehensive Cancer Center, Georgetown University, Washington, DC, USA; ${ }^{2}$ Department of Internal Medicine, Medstar Washington \\ Hospital Center, Washington DC, USA \\ Contributions: (I) Conception and design: All authors; (II) Administrative support: All authors; (III) Provision of study materials or patients: All \\ authors; (IV) Collection and assembly of data: All authors; (V) Data analysis and interpretation: All authors; (VI) Manuscript writing: All authors; (VII) \\ Final approval of manuscript: All authors. \\ Correspondence to: Stephen V. Liu, MD. Lombardi Comprehensive Cancer Center, Georgetown University Hospital, 3800 Reservoir Road NW, \\ Washington DC 20007, USA. Email: stephen.v.liu@gunet.georgetown.edu.
}

\begin{abstract}
Small cell lung cancer (SCLC) is a particularly lethal subtype of lung cancer whose treatment landscape has been relatively devoid of advance. The recent integration of immunotherapy in the firstline treatment of SCLC has improved overall survival (OS), prompting the first major paradigm shift for this disease in decades. Despite this improvement in outcomes, most patients with SCLC will relapse after initial response. Standard salvage systemic therapy for SCLC remains disappointing, with few approved agents and consistently poor outcomes. The need for novel agents to combat this disease remains pressing. Fortunately, there are several agents in various stages of development that hold potential as novel treatments for advanced SCLC. Lurbinectedin, which targets active transcription, has shown activity in platinumsensitive and platinum-resistant SCLC as monotherapy and in combination with doxorubicin. Aurora A kinase (AAK) inhibitors showed initial activity when given with paclitaxel but in randomized studies, failed to improve outcomes over paclitaxel plus placebo. However, in the subset of patients with MYC expression, targeting AAK was effective. Similarly, agents targeting poly-ADP ribose (PARP) pair well with other DNA damaging drugs but in the subset of patients whose tumors express Schlafen-11 (SLFN-11), efficacy appeared greater. CDK 4/6 inhibition is being explored, primarily as a means to protect myeloid cells during cytotoxic chemotherapy in a strategy expected to be uniquely effective in SCLC. Ongoing trials are also studying are novel formulations of established cytotoxic agents. Delta-like protein 3 (DLL3) is an appealing therapeutic target given its selective expression on SCLC cells, but after initial exciting results, the antibodydrug conjugate (ADC) Rovalpituzumab tesirine (Rova-T) did not have a favorable efficacy to toxicity profile in randomized trials. Other agents targeting DLL3 are under study. Targeting angiogenesis has yielded modest improvements in the past but newer agents such as anlotinib are renewing interest. While the current therapeutic landscape beyond chemo-immunotherapy remains the same as it was decades ago, drug development for SCLC is rapidly moving forward and promises to deliver the needed novel agents in the very near future.
\end{abstract}

Keywords: Small cell lung cancer (SCLC); lurbinectedin; poly-ADP ribose (PARP); Delta-like protein 3 (DLL3); Aurora A kinase (AAK); CDK 4/6

Submitted Apr 27, 2020. Accepted for publication May 13, 2020.

doi: $10.21037 /$ jtd-2019-sclc-10

View this article at: http://dx.doi.org/10.21037/jtd-2019-sclc-10 


\section{Introduction}

Small cell lung cancer (SCLC) is an aggressive neuroendocrine malignancy with a high proliferative index that accounts for just under $15 \%$ of new lung cancer diagnoses (1). While less common than non-small cell lung cancer (NSCLC), SCLC is disproportionately lethal. The majority of patients with SCLC present with advanced disease where systemic therapy is the primary treatment modality. The historic standard of platinumdoublet chemotherapy provides consistently high response rates (RR), but while initially responsive to numerous cytotoxic agents or radiotherapy, SCLC is characterized by a rapid and near-inevitable relapse (2). The addition of immunotherapy to first-line chemotherapy has provided a long-awaited improvement in SCLC outcomes. Two randomized phase III trials have demonstrated that the addition of an anti-PD-L1 antibody to platinum doublet chemotherapy (atezolizumab in IMpower 133 and durvalumab in CASPIAN) significantly improves both progression free survival (PFS) and overall survival (OS), establishing new standards of care $(3,4)$.

While there is a clear survival benefit seen with early integration of immunotherapy, most patients with SCLC will relapse. Active salvage therapy is an ever-relevant clinical need, particularly in light of the suboptimal outcomes with standard treatment. The only second-line agent currently approved by the US FDA is topotecan. Topotecan, though, has limited efficacy, with a RR of $24 \%$ and a survival of about 6 months. These modest outcomes are accompanied by notable toxicity including relatively high rates of grade $3-4$ thrombocytopenia (10\%), grade 3-4 anemia (18\%) and grade 4 neutropenia (38\%) (5). Despite the need for novel therapeutics, there has been a striking lack of new approved agents for the treatment of relapsed SCLC. There are many challenges to drug development in SCLC including the rapid clinical course of the disease, the comorbidities that accompany this smoking-related malignancy, and poor access to adequate tissue samples for analysis to guide rational drug design. Unlike NSCLC, targeted therapy has had no impact on the treatment of SCLC. While the typical SCLC genome harbors many genomic aberrations, there is a notable absence of actionable alterations in protein kinases, the likes of which have transformed the therapeutic landscape of NSCLC. Rather, SCLC is characterized by loss-of-function alterations in tumor suppressor genes TP53 and RB1; the multitude of mutations seen in the SCLC genome have not yielded viable drug targets to date (6).

The current therapeutic landscape for relapsed SCLC is bleak. Fortunately, a greater understanding of the biology of SCLC and the underlying heterogeneity of the disease have provided promising inroads in establishing novel therapeutic strategies.

\section{Lurbinectedin}

One of the agents relatively far in development is lurbinectedin, an RNA polymerase II inhibitor that targets active transcription. Lurbinectedin can have a direct cytotoxic effect, inducing apoptosis in constitutively active tumor cells, but it may also impact the tumor microenvironment but targeting tumor associated macrophages (7). A phase I study determined safe dosing schedules for lurbinectedin monotherapy of $4 \mathrm{mg} / \mathrm{m}^{2}$ or, because clearance was independent of body surface area, a flat dose of $7 \mathrm{mg}$ intravenously (IV) every 3 weeks (8). Another phase I combination study, based on preclinical synergy, established tolerability of lurbinectedin $4 \mathrm{mg}$ (flat dose) with doxorubicin $\left(50 \mathrm{mg} / \mathrm{m}^{2}\right)$ in 21-day cycles (9). This combination was studied in 12 patients with platinumsensitive relapsed SCLC (defined as a platinum-free interval of at least 90 days) who had received only one prior line of therapy and showed promise. Lurbinectedin plus doxorubicin offered RR of $92 \%$ with a median PFS of 5.8 months. In 9 patients with platinum-resistant relapsed SCLC (platinum-free interval under 90 days) who had received only one prior line of therapy, there was still activity, with a RR of $33 \%$ and a median PFS of 3.5 months. Toxicities were primarily hematologic in nature, with high rates of grade 3-4 neutropenia (95\%), leukopenia (79\%), anemia (47\%) and thrombocytopenia (26\%).

A phase II basket study explored the activity of lurbinectedin $3.2 \mathrm{mg} / \mathrm{m}^{2}$ monotherapy (given every 21 days) in various solid tumors, including a 105 -patient cohort with relapsed SCLC that had received one prior line of therapy (10). This study confirmed activity with an investigator-assessed RR of $35.2 \%$ and a median duration of response (DOR) of 5.3 months. The median OS was 9.3 months. In 60 patients with platinum-sensitive relapse (platinum-free interval of at least 90 days), the RR was $45 \%$ with a DOR of 6.2 months. In patients with platinumresistant SCLC (platinum-free interval shorter than 90 days), there was still activity; the RR was $22.2 \%$ with a DOR of 4.7 months. Grade 3-4 adverse events were again primarily hematologic: grade 3-4 neutropenia was observed in $46.5 \%$ 
of patients. ATLANTIS is a randomized phase III trial of lurbinectedin $2 \mathrm{mg} / \mathrm{m}^{2}$ (capped at $4 \mathrm{mg}$ ) plus doxorubicin $40 \mathrm{mg} / \mathrm{m}^{2}$ every 3 weeks versus standard chemotherapy (topotecan or cyclophosphamide plus doxorubicin plus vincristine) in patients with relapsed SCLC (11). The dosing schedule was modified from the previous phase Ib study to limit myelosuppression in a less selective, previously treated patient population. Results from ATLANTIS are pending.

\section{Bcl-2 Inhibition}

Bcl-2 is an inhibitor of apoptosis often overexpressed in SCLC and another appealing therapeutic target (12). The small molecule Bcl-2 inhibitor navitoclax was explored in a single-arm, phase I/II study that included 39 patients with relapsed SCLC (13). Patients received navitoclax $150 \mathrm{mg}$ daily for 7 days followed by $325 \mathrm{mg}$ daily thereafter in 21-day cycles. Only one patient achieved a response (RR $2.6 \%$ ) with $23 \%$ of patients achieving stable disease. The median PFS was 1.5 months and median OS was 3.2 months. The most common adverse event was thrombocytopenia, with any grade noted in $62 \%$ of patients and $41 \%$ of patients experiencing grade 3-4 thrombocytopenia, though bleeding complications were not observed.

With limited monotherapy efficacy, combinations are being explored. Preclinical models noted downregulation of HIF-1a gene sets in response to Bcl-2 inhibition (14). Rapamycin, an mTOR inhibitor, regulates the HIF-1a pathway and preclinically, when combined with a Bcl-2 inhibitor, prevented resistance and was highly synergistic in SCLC models. An ongoing clinical trial of navitoclax and vistusertib is exploring this synergistic relationship (NCT 03366103).

\section{Aurora A kinase (AAK) inhibition}

AAK is a regulator of mitosis that is overexpressed in SCLC (15). AAK binds directly with $c-M Y C$, a transcription factor amplified and overexpressed in $18-31 \%$ of SCLC samples (16). Disruption of the AAK and c-MYC complex with AAK inhibitors induces apoptosis and represents an appealing therapeutic strategy. Alisertib is an orally bioavailable AAK inhibitor with observed clinical activity in SCLC. A 5-arm phase II trial of alisertib in various malignancies included 60 patients with relapsed SCLC, 48 of whom were evaluable for response (17). Alisertib was given orally at a dose of $50 \mathrm{mg}$ twice daily for 7 out of every 21 days. The RR in the SCLC cohort was $21 \%$ with a median DOR of 4.1 months and a median PFS of 2.1 months. There were 36 patients with platinum-sensitive relapsed SCLC (with a platinum-free interval of at least 90 days) and 12 patients with platinum-resistant relapse. In the platinumsensitive group, RR was $19 \%$, DOR was 3.1 months and median PFS was 2.6 months. In the platinum-refractory group, RR was $25 \%$, DOR was 4.3 months and median PFS was 1.7 months. Drug-related adverse events were largely hematologic and in the SCLC cohort, included a $37 \%$ incidence of grade 3-4 neutropenia, $12 \%$ grade 3-4 anemia, $8 \%$ grade $3-4$ thrombocytopenia and $5 \%$ grade $3-4$ fatigue.

A subsequent phase II trial randomized 178 patients with relapsed SCLC to paclitaxel plus alisertib or placebo (18). The dosing of alisertib was adjusted in light of overlapping toxicities with paclitaxel chemotherapy. Alisertib was administered orally at $40 \mathrm{mg}$ twice daily on days $1-3$, $8-10$ and $15-17$ in 3-week cycles with paclitaxel $60 \mathrm{mg} / \mathrm{m}^{2}$ given IV weekly on days 1,8 and 15 . In the placebo arm, paclitaxel was given at a dose of $80 \mathrm{mg} / \mathrm{m}^{2}$ on the same schedule. In the intention-to-treat population, alisertib did not significantly improve outcomes over placebo. The median PFS in the alisertib/paclitaxel arm was 3.32 months compared to 2.17 months with placebo/paclitaxel $(\mathrm{P}=0.113)$. In patients with platinum-sensitive relapse (using a 90-day platinum-free interval threshold), the median PFS was 3.72 months with alisertib and 3.34 months with placebo $(\mathrm{P}=0.590)$ whereas in patients with resistant relapse, the median PFS was 2.86 months with alisertib and 1.68 months with placebo $(\mathrm{P}=0.037)$. There was no survival advantage observed with alisertib. Median OS was 6.86 months with alisertib versus 5.58 months with placebo (HR 0.93; 95\% CI: 0.652-1.341, $\mathrm{P}=0.714)$. RR was similar between alisertib and placebo (22\% versus $18 \%$ ) as was DOR (3.16 and 2.79 months, respectively).

Exploratory biomarker analyses from this study identified an important potential path forward for use of AAK inhibitors in relapsed SCLC. Because this strategy targets the MYC-AAK complex, expression of c-MYC is a potential predictive marker for AAK inhibition. In the randomized trial of paclitaxel plus placebo versus paclitaxel plus alisertib, c-MYC expression by immunohistochemistry was performed on tissue samples from 46 patients; 33 of these $(72 \%)$ were positive while $13(28 \%)$ were negative. In patients with c-MYC positive SCLC, outcomes did favor alisertib over placebo: median PFS was 4.64 months with alisertib and paclitaxel compared to 2.27 months with placebo and paclitaxel (HR 0.29; 95\% CI: 0.12-0.72). In tumors that did not express c-MYC, outcomes favored the 
placebo arm (with the higher dose of paclitaxel): median PFS was 3.32 months with alisertib plus paclitaxel versus 5.16 months with placebo plus paclitaxel (HR 11.8; 95\% CI: 1.52-91.2). Drug development in relapsed SCLC will need to incorporate the use of rational biomarkers and MYC expression represents a potentially powerful tool to guide use of AAK inhibition going forward. Additional predictors are being explored including phosphorylation of $\mathrm{Rb}$ (19). Loss of phosphorylated RB led to a hyperdependence on Aurora kinase in animal models, supporting its study as a potential predictor, though given the high prevalence of $\mathrm{Rb}$ loss in SCLC, its potential clinical role is unclear.

\section{Poly-ADP ribose (PARP) inhibition}

PARP enzymes are responsible for detection and baseexcision repair of single-strand DNA breaks, and given their high expression in SCLC, PARP has been a target of interest for drug development (20). Talazoparib was evaluated in a phase I trial with a 23-patient expansion part for patients with relapsed SCLC (21). Patients received talazoparib $1.0 \mathrm{mg}$ daily but only 2 patients (9\%) achieved a partial response. The median PFS was 11.1 weeks. Development then moved beyond PARP inhibitor monotherapy.

Rational strategies sought to combine PARP inhibitors with DNA-damaging agents, namely, cytotoxic chemotherapy. The addition of the PARP inhibitor veliparib, however, did not improve outcomes when added to first-line chemotherapy. A phase I trial established tolerability of the combination in 9 patients with SCLC (22). Based on this experience, in the phase II ECOG-ACRIN 2511 trial, 128 patients with treatment-naïve, advanced SCLC were treated with four 21-day cycles of cisplatin $75 \mathrm{mg} / \mathrm{m}^{2}$ (day 1) plus etoposide $100 \mathrm{mg} / \mathrm{m}^{2}$ (days 1-3) and were randomized 1:1 to receive concurrent veliparib $100 \mathrm{mg}$ or placebo orally twice daily (days 1-7) (23). PFS, the primary endpoint, was similar between the veliparib arm (median 6.1 months) and the placebo arm (median 5.5 months). Median OS was 10.3 months with veliparib plus chemotherapy and 8.9 months with placebo plus chemotherapy $(\mathrm{P}=0.17)$. There was no difference in $\mathrm{RR}$ ( $71.9 \%$ for veliparib, $65.6 \%$ for placebo; $\mathrm{P}=0.29$ ) but the addition of veliparib compared to placebo did increase the rate of some toxicities, including grade 3 lymphopenia ( $8 \%$ versus $0 \%$ ) and grade $3-4$ neutropenia (49\% versus $32 \%$ ).

In patients with previously treated SCLC, other PARP combinations showed promise. A phase II trial explored the oral alkylating agent temozolomide given with either veliparib $40 \mathrm{mg}$ twice daily or placebo (24). In 104 patients, responses were more frequent with the addition of veliparib (RR 39\% versus $14 \%, \mathrm{P}=0.16$ ). However, this did not translate into a difference in 4-month PFS rate $36 \%$ with veliparib, $27 \%$ with placebo, $\mathrm{P}=0.19)$ or median OS $(8.2$ versus 7.0 months). Veliparib did increase toxicity including higher rates of grade 3-4 thrombocytopenia (50\% versus $9 \%$ ) and grade $3-4$ neutropenia (31\% versus $7 \%$ ) when compared to the temozolomide with placebo. Comparable outcomes were seen in a single arm phase I/II study of the PARP inhibitor olaparib plus temozolomide (25). The phase I portion established a schedule of temozolomide $75 \mathrm{mg} / \mathrm{m}^{2}$ daily with olaparib $200 \mathrm{mg}$ orally twice daily, both given on days 1-7 in 21-day cycles. In 48 evaluable patients with previously treated SCLC, the RR was $41.7 \%$ with a median PFS of 4.2 months and a median OS of 8.5 months. Among the 34 patients with platinum-sensitive relapse (using a 90-day threshold), the RR was $47.1 \%$ compared to $28.6 \%$ in the 14 patients with platinum-resistant relapse. Median PFS and median OS were 4.5 and 9.4 months for sensitive relapse and 2.9 and 7.4 months for resistant relapse. There was not a statistically significant difference between platinum-sensitive and platinum-resistant patients in PFS (HR 0.76, $\mathrm{P}=0.400$ ) or OS (HR 1.05, $\mathrm{P}=0.898$ ), though the sample sizes were limited. There seems to be activity with PARP inhibitor combinations featuring cytotoxic chemotherapy, but they have an unclear role at this time. It is likely that a subset of patients is deriving most of the benefit and efforts to enrich study populations may provide stronger signals.

A promising predictive biomarker for PARP inhibitors in SCLC is expression of Schlafen-11 (SLFN-11), which mediates response to DNA damage and replication stress. SLFN-11 expression by immunohistochemistry was correlated with outcomes in the randomized phase II study of temozolomide plus veliparib versus temozolomide plus placebo (Table 1) (24). In this trial, the addition of veliparib to temozolomide improved outcomes in the SLFN-11positive subset. In patients receiving temozolomide and veliparib, outcomes were superior in SLFN-11-positive cases compared to SLFN-11 negative, with longer median PFS (5.7 versus 3.6 months, $\mathrm{P}=0.009$ ) and median $\mathrm{OS}$ (12.2 versus 7.5 months, $\mathrm{P}=0.014$ ). In patients receiving temozolomide plus placebo, there was no difference in median PFS $(\mathrm{P}=0.162)$ or OS $(\mathrm{P}=0.634)$ on the basis of SLFN-11 expression. Prospective studies in patients with SLFN-11-positive SCLC are underway and in development. 
Table 1 OS and PFS (both measured in months) with TMZ and either veliparib or placebo in the overall study population and by presence or absence of SLFN-11 expression by immunohistochemistry (20)

\begin{tabular}{|c|c|c|c|c|c|c|}
\hline Outcome & \multicolumn{2}{|c|}{ Overall } & \multicolumn{2}{|c|}{ SLFN-11 positive } & \multicolumn{2}{|c|}{ SLFN-11 negative } \\
\hline PFS & 3.8 & 2.0 & 5.7 & 1.8 & 3.6 & 3.6 \\
\hline os & 8.2 & 7.0 & 12.2 & 9.4 & 7.5 & 7.7 \\
\hline
\end{tabular}

OS, overall survival; PFS, progression free survival; TMZ, temozolomide; SLFN-11, Schlafen-11.

In addition, exploratory analysis of the outcomes by SLFN11 expression in the phase II ECOG-ACRIN 2511 trial of cisplatin, etoposide and veliparib are ongoing and could influence any potential role for this triplet in the future.

An intriguing role for PARP inhibitors in the treatment of SCLC is as an immunomodulator. Preclinical studies demonstrate an upregulation of PD-L1 expression in response to olaparib and enhancement of both T-cell infiltration and tumor regression (26). This appears to be mediated by the STING innate immune pathway. This effect has yet to be validated clinically; in a phase II study of olaparib and the PD-L1 inhibitor durvalumab, responses were noted in only $10.5 \%$ of patients (27).

\section{Cyclin dependent kinase 4/6 (CDK 4/6)}

Inhibition of $\mathrm{CDK} 4 / 6$ prevents phosphorylation of $\mathrm{Rb}$ and leads to an arrest in the cell cycle at G1. This has been an effective therapeutic strategy in some malignancies, such as breast cancer, where CDK 4/6 inhibitors are effective and approved treatments. They may play a different role in the treatment of SCLC. One of the characteristic genomic findings of SCLC is loss of Rb (6); thus, CDK 4/6 inhibitors would be expected to have minimal impact on the cell cycle of SCLC cells. Trilaciclib was studied in patients with SCLC, but not as an anti-tumor agent, rather as a myeloprotective agent. When administered with cytotoxic chemotherapy, trilaciclib could induce G1 cell cycle arrest in host hematopoietic stem and progenitor cells, with relatively little impact on the anti-tumor effect of chemotherapy. Several trials explored the benefit of adding trilaciclib to chemotherapy as a means to reduce the myelosuppressive effects. Three separate phase II studies explored this strategy in SCLC: trilaciclib with first-line carboplatin plus etoposide (G1T28-02), trilaciclib with firstline carboplatin plus etoposide with atezolizumab (G1T2805), and trilaciclib with second-line topotecan (G1T28-03) (28-30). All of these studies met their primary endpoints for significant reductions in the incidence and duration of neutropenia. As expected, the efficacy of chemotherapy did not appear to be impaired and patient reported outcomes were improved with the addition of trilaciclib. Preclinical studies have also analyzed the effect of CDK 4/6 inhibition on immune cell function (31). After short term exposure to CDK 4/6 inhibition, $T$ cell proliferation was decreased but effector $\mathrm{T}$ cell activation and tumor infiltration were enhanced.

\section{Novel formulations of chemotherapy}

Many cytotoxic agents have demonstrated limited efficacy in relapsed SCLC, but their use can be limited by toxicity. Newer formulations of traditional cytotoxic agents have renewed interest in chemotherapy-based approaches. For example, nano-particle albumin bound (nab)-paclitaxel has emerged as a well-tolerated and efficacious agent for the treatment of breast cancer, pancreatic cancer and NSCLC (32). The single-arm phase II NABSTER trial studied the efficacy of nab-paclitaxel in 68 patients with relapsed SCLC (33). Using a 60-day platinum-free interval to define platinum sensitivity, 43 patients had platinumsensitive relapse and 25 patients had platinum-refractory relapse. Patients received nab-paclitaxel monotherapy weekly on days 1, 8 and 15 in 28-day cycles. Treatment was well tolerated; the only grade 3-4 toxicity observed was neutropenia (9\%). Reports of efficacy are pending.

A nanoliposomal formulation of irinotecan was developed to minimize serum exposure and allow for delivery through leaky tumor vasculature where macrophages activate the drug (34). Preclinical models suggest high SLFN11 expression may help predict efficacy of nanoliposomal irinotecan (nal-IRI). When given intravenously $70 \mathrm{mg} / \mathrm{m}^{2}$ every 2 weeks, nal-IRI, early efficacy suggested a RR in $33.3 \%$ of patients (4 out of 12 ), though more complete data are still pending (35).

For patients with platinum-sensitive relapse, retreatment 
with platinum plus etoposide has been an effective treatment option (36). Novel strategies to re-sensitize SCLC tumors to platinum-based chemotherapy are emerging. $\mathrm{RRx}-001$ is a dinitroazetidine derivative that lists among its effects epigenetic modulation and induction of the M1 macrophage phenotype (37). A single-arm phase II explored treatment with $\mathrm{RRx}-001$ followed by re-challenge with platinum plus etoposide in patients with relapsed SCLC. The trial enrolled 26 patients, 19 of whom (73.1\%) had platinumresistant relapse. The $\mathrm{RR}$ to $\mathrm{RRx}-001$ was $3.8 \%$ (1 patient) and the RR to the subsequent platinum plus etoposide was $26.9 \%$ including one complete response. The median OS was 8.6 months with a 12 -month OS rate of $44.1 \%$. In the subset of patients with platinum-resistant disease treated in the third-line setting or later $(n=14)$, the RR was $21.4 \%$ with a median PFS of 5.8 months and a median OS of 8.6 months. RRx-001 was well tolerated with the most common treatment emergent adverse events being infusion site reaction $(23 \%)$, decreased appetite $(15.3 \%)$ and headache $(11.5 \%)$.

\section{Delta-like protein 3 (DLL3)}

Another promising target for drug development in SCLC is DLL3. DLL3 is a member of the NOTCH receptor ligand family that inhibits NOTCH activation. DLL3 is expressed in about $80 \%$ of SCLC specimens with low rates of expression in normal adult tissue (38). Rovalpituzumab tesirine (Rova-T) is an antibody-drug conjugate (ADC) that links a DLL3 specific IgG1 monoclonal antibody with a potent DNA cross-linking agent. A phase I study of Rova-T in patients with relapsed SCLC showed initial promise. Given intravenously at a dose of $0.3 \mathrm{mg} / \mathrm{kg}$ every 6 weeks, the initial reported RR was $18 \%$ with a median DOR of 5.6 months. Median PFS was 3.1 months and median OS was 4.6 months. Grade 3 or higher toxicities included thrombocytopenia (11\%), pleural effusion (8\%) and lipase elevation (7\%).

While initial activity was encouraging, the subsequent randomized trials of Rova- $\mathrm{T}$ failed to establish a role for Rova-T in the treatment of SCLC. TRINITY was a singlearm phase II study of third-line (and beyond) Rova- $T$ in 339 patients with SCLC positive for DLL3 expression by immunohistochemistry (39). Rova-T offered a modest RR of $12.4 \%$ and a median OS of 5.6 months. Toxicity was significant, as grade 3-5 treatment emergent adverse events were seen in $63 \%$ of patients; grade 5 events occurred in $10 \%$ of patients. Drug-related serious treatment emergent adverse events were reported in $30 \%$ of patients. TAHOE and MERU were both randomized phase III trials of Rova-T in the second line and maintenance settings, respectively (40). Both studies were terminated early for failure to meet interim primary PFS and OS endpoints. Limited activity with higher than expected rates of toxicity have led to the discontinuation of Rova-T development.

Rova-T is no longer under development, but DLL3 remains an appealing target. Other molecules are being developed to exploit the selective expression of DLL3 on SCLC tumor cells. AMG 757 is a rationally designed, bispecific $\mathrm{T}$ cell engager $\left(\mathrm{BiTE}^{\circledR}\right)$ designed to target and crosslink DLL3 on SCLC cells with CD3-positive T cells, facilitating $\mathrm{T}$ cell-mediated tumor lysis (41). Preclinical studies demonstrate effective induction of cell death in DLL3-expressing cancer cells with growth inhibition in SCLC xenograft models $(42,43)$. A phase I trial of AMG 757 in patients with relapsed SCLC is underway. AMG 119 employs an alternate strategy whereby autologous $\mathrm{T}$ cells are genetically modified ex vivo to express transmembrane chimeric antigen receptor targeting DLL3 (44). Preclinical data show high potency and specificity for tumor cells expressing DLL3; this agent is also in phase I investigation in patients with relapsed SCLC.

\section{Fibroblast growth factor receptor (FGFR)}

FGFR1 amplifications have been observed infrequently in SCLC specimens (6). Exploration of FGFR1 as a therapeutic target in SCLC has been limited, in part due to challenges with rapid next-generation sequencing (NGS) of typically scant SCLC specimens relative to the aggressive disease course. One report, though, showed efficacy of this strategy. A patient treated with platinum doublet chemotherapy followed by second line topotecan was found to have FGFR1 amplification after plasma-based NGS (45). Pazopanib is a commercially available multi-kinase inhibitor with activity at FGFR1. She received pazopanib $800 \mathrm{mg}$ orally once daily off-label and achieved a partial response after 2 months, continuing therapy for 12 months overall.

\section{Angiogenesis}

Agents targeting angiogenesis have been studied in SCLC with mixed results. Vascular endothelial growth factor (VEGF) plays a central role in angiogenesis and high VEGF levels are a poor prognostic factor in SCLC (46). Bevacizumab is a monoclonal antibody targeting VEGF. 
The addition of bevacizumab to chemotherapy did improve PFS but did not have an impact on OS (47). Sunitinib is a multi-kinase tyrosine kinase inhibitor that also targets VEGF. Use of sunitinib as maintenance therapy after chemotherapy had a similar impact: longer PFS but no improvement in OS (48).

Anlotinib is another multi-kinase inhibitor with activity at VEGF receptors 2 and 3 as well as other potentially relevant receptors such as FGFR 1-4, platelet-derived growth factors $\alpha$ and $\beta$, and stem cell-factor receptor (c-kit) (49). ALTER 1202 was a double-blind, randomized phase II trial that included 120 patients with SCLC who had received 2 prior lines of chemotherapy (50). Patients were randomized 2:1 to receive anlotinib $12 \mathrm{mg}$ orally or placebo once daily for 14 days in 21-day cycles. Anlotinib improved median PFS to 4.1 months from 0.7 months with placebo (HR 0.19; 95\% CI: 0.12-0.32). There was also an improvement in OS. Median survival in the 82 patients treated with anlotinib was 7.3 months and in the 38 patients receiving placebo, median OS was 4.9 months (HR 0.53; $95 \%$ CI: $0.3-0.8, \mathrm{P}=0.0029)$. The 1 -year survival rate was $30.6 \%$ compared to $13.1 \%$ in the control arm.

\section{Conclusions}

Drug development in SCLC has been remarkably challenging, in part due to its aggressive biology, typical patient characteristics and numerous practical challenges. The lack of new drug approvals does not reflect the lack of interest; dozens of trials have simply failed to improve outcomes. Immunotherapy has provided a brief respite from the long drought of new approvals, changing the paradigm initially in the third-line setting and ultimately in the first-line setting. For patients who relapse after chemoimmunotherapy, standard therapy remains starkly limited. There is reason for optimism, though, as several agents have shown promise and will likely impact this disease in the relatively near future. The likely path forward for drug development in SCLC will be contingent on the development of rational and reliable predictive biomarkers. While NGS may occasionally yield viable therapeutic targets, alternative testing strategies will be needed.

There is a growing appreciation that SCLC, while histologically uniform, is an incredibly heterogeneous disease. There are distinct molecular subtypes of SCLC classified on the basis of expression of transcriptional regulators (51). Delivery of specific agents to patients with specific subtypes of SCLC is likely to yield superior results.
Using expression of SLFN-11 or MYC, as outlined above, is a better path forward than empiric drug exploration in an unselected population, which has been the standard strategy for decades. Obtaining adequate tissue specimens for analysis has historically been challenging in SCLC but the only rational drug development strategy in SCLC will require rational subclassification. Once achieved, many of the agents that have failed in SCLC may need to be revisited as the failure may not have been with the drug, but rather the patient population studied. With trials such as this already planned, there will likely be significant gains in this setting in the years to come.

\section{Acknowledgments}

Funding: None.

\section{Footnote}

Provenance and Peer Review: This article was commissioned by the Guest Editor (Jose Pacheco) for the series "Small Cell Lung Cancer" published in Fournal of Thoracic Disease. The article was sent for external peer review organized by the Guest Editor and the editorial office.

Conflicts of Interest: All authors have completed the ICMJE uniform disclosure form (available at: http://dx.doi. org/10.21037/jtd-2019-sclc-10). The series "Small Cell Lung Cancer" was commissioned by the editorial office without any funding or sponsorship. SVL reports grants from Alkermes, grants, personal fees and non-financial support from AstraZeneca, grants from Bayer, non-financial support from Boehringer-Ingelheim, grants from Blueprint, grants and personal fees from Bristol-Myers Squibb, personal fees from Catalyst, personal fees from Celgene, grants from Corvus, personal fees from G1 Therapeutics, grants, personal fees and non-financial support from Genentech/Roche, personal fees from Guardant Health, personal fees from Janssen, personal fees from Lilly, personal fees from LOXO, grants, personal fees and nonfinancial support from Merck/MSD, grants from Merus, grants from Molecular Partners, grants and personal fees from Pfizer, personal fees from PharmaMar, grants from Rain Therapeutics, grants from RAPT, personal fees from Regeneron, grants from Spectrum, personal fees from Takeda, grants from Turning Point Therapeutics, outside the submitted work. The authors have no other conflicts of interest to declare. 
Ethical Statement: The authors are accountable for all aspects of the work in ensuring that questions related to the accuracy or integrity of any part of the work are appropriately investigated and resolved.

Open Access Statement: This is an Open Access article distributed in accordance with the Creative Commons Attribution-NonCommercial-NoDerivs 4.0 International License (CC BY-NC-ND 4.0), which permits the noncommercial replication and distribution of the article with the strict proviso that no changes or edits are made and the original work is properly cited (including links to both the formal publication through the relevant DOI and the license). See: https://creativecommons.org/licenses/by-nc-nd/4.0/.

\section{References}

1. Siegel RL, Miller KD, Jemal A. Cancer statistics, 2019. CA Cancer J Clin 2019;69:7-34.

2. Armstrong SA, Liu SV. Immune Checkpoint Inhibitors in Small Cell Lung Cancer: A Partially Realized Potential. Adv Ther 2019;36:1826-32.

3. Horn L, Mansfield AS, Szczesna A, et al. First-Line Atezolizumab plus Chemotherapy in Extensive-Stage Small-Cell Lung Cancer. N Engl J Med 2018;379:2220-9.

4. Paz-Ares L, Dvorkin M, Chen Y, et al. Durvalumab plus platinum-etoposide versus platinum-etoposide in firstline treatment of extensive-stage small-cell lung cancer (CASPIAN): a randomised, controlled, open-label, phase 3 trial. Lancet 2019;394:1929-39.

5. von Pawel J, Schiller JH, Shepherd FA, et al. Topotecan versus cyclophosphamide, doxorubicin, and vincristine for the treatment of recurrent small-cell lung cancer. J Clin Oncol 1999;17:658-67.

6. George J, Lim JS, Jang SJ, et al. Comprehensive genomic profiles of small cell lung cancer. Nature 2015;524:47-53.

7. Santamaría Nuñez G, Robles CM, Giraudon C, et al. Lurbinectedin Specifically Triggers the Degradation of Phosphorylated RNA Polymerase II and the Formation of DNA Breaks in Cancer Cells. Mol Cancer Ther 2016;15:2399-412.

8. Elez ME, Tabernero J, Geary D, et al. First-in-human phase I study of Lurbinectedin (PM01183) in patients with advanced solid tumors. Clin Cancer Res 2014;20:2205-14.

9. Calvo E, Moreno V, Flynn M, et al. Antitumor activity of lurbinectedin (PM01183) and doxorubicin in relapsed small-cell lung cancer: results from a phase I study. Ann Oncol 2017;28:2559-66.
10. Trigo J, Subbiah V, Besse B, et al. Lurbinectedin as secondline treatment for patients with small-cell lung cancer: a single-arm, open-label, phase 2 basket trial. Lancet Oncol 2020;21:645-54.

11. Farago AF, Drapkin BJ, Lopez-Vilarino de Ramos JA, et al. ATLANTIS: a Phase III study of lurbinectedin/ doxorubicin versus topotecan or cyclophosphamide/ doxorubicin/vincristine in patients with small-cell lung cancer who have failed one prior platinum-containing line. Future Oncol 2019;15:231-9.

12. Jiang SX, Sato Y, Kuwao S, et al. Expression of bcl2 oncogene protein is prevalent in small cell lung carcinomas. J Pathol 1995;177:135-8.

13. Rudin CM, Hann CL, Garon EB, et al. Phase II study of single-agent navitoclax (ABT-263) and biomarker correlates in patients with relapsed small cell lung cancer. Clin Cancer Res 2012;18:3163-9.

14. Gardner EE, Connis N, Poirier JT, et al. Rapamycin rescues ABT-737 efficacy in small cell lung cancer. Cancer Res 2014;74:2846-56.

15. Barr AR, Gergely F. Aurora-A: the maker and breaker of spindle poles. J Cell Sci 2007;120:2987-96.

16. Kim YH, Girard L, Giacomini CP, et al. Combined microarray analysis of small cell lung cancer reveals altered apoptotic balance and distinct expression signatures of MYC family gene amplification. Oncogene 2006;25:130-8.

17. Melichar B, Adenis A, Lockhart AC, et al. Safety and activity of alisertib, an investigational aurora kinase A inhibitor, in patients with breast cancer, small-cell lung cancer, non-small-cell lung cancer, head and neck squamous-cell carcinoma, and gastro-oesophageal adenocarcinoma: a five-arm phase 2 study. Lancet Oncol 2015;16:395-405.

18. Owonikoko TK, Niu H, Nackaerts K, et al. Randomized Phase II Study of Paclitaxel plus Alisertib versus Paclitaxel plus Placebo as Second-Line Therapy for SCLC: Primary and Correlative Biomarker Analyses. J Thorac Oncol 2020;15:274-87.

19. Oser MG, Fonseca R, Chakraborty AA, et al. Cells Lacking the RB1 Tumor Suppressor Gene Are Hyperdependent on Aurora B Kinase for Survival. Cancer Discov 2019;9:230-47.

20. Byers LA, Wang J, Nilsson MB, et al. Proteomic profiling identifies dysregulated pathways in small cell lung cancer and novel therapeutic targets including PARP1. Cancer Discov 2012;2:798-811.

21. de Bono J, Ramanathan RK, Mina L, et al. Phase I, Dose-Escalation, Two-Part Trial of the PARP Inhibitor 
Talazoparib in Patients with Advanced Germline BRCA1/2 Mutations and Selected Sporadic Cancers. Cancer Discov 2017;7:620-9.

22. Owonikoko TK, Dahlberg SE, Khan SA, et al. A phase 1 safety study of veliparib combined with cisplatin and etoposide in extensive stage small cell lung cancer: A trial of the ECOG-ACRIN Cancer Research Group (E2511). Lung Cancer 2015;89:66-70.

23. Owonikoko TK, Dahlberg SE, Sica GL, et al. Randomized Phase II Trial of Cisplatin and Etoposide in Combination With Veliparib or Placebo for Extensive-Stage Small-Cell Lung Cancer: ECOG-ACRIN 2511 Study. J Clin Oncol 2019;37:222-9.

24. Pietanza MC, Waqar SN, Krug LM, et al. Randomized, Double-Blind, Phase II Study of Temozolomide in Combination With Either Veliparib or Placebo in Patients With Relapsed-Sensitive or Refractory Small-Cell Lung Cancer. J Clin Oncol 2018;36:2386-94.

25. Farago AF, Yeap BY, Stanzione M, et al. Combination Olaparib and Temozolomide in Relapsed Small-Cell Lung Cancer. Cancer Discov 2019;9:1372-87.

26. Sen T, Rodriguez BL, Chen L, et al. Targeting DNA Damage Response Promotes Antitumor Immunity through STING-Mediated T-cell Activation in Small Cell Lung Cancer. Cancer Discov 2019;9:646-61.

27. Thomas A, Vilimas R, Trindade C, et al. Durvalumab in Combination with Olaparib in Patients with Relapsed SCLC: Results from a Phase II Study. J Thorac Oncol 2019;14:1447-57.

28. Daniel D, Kuchava V, Bondarenko I, et al. Trilaciclib (T) decreases myelosuppression in extensive-stage small cell lung cancer (ES-SCLC) patients receiving first-line chemotherapy plus atezolizumab. Ann Oncol 2019;30:v713.

29. Weiss JM, Csoszi T, Maglakelidze M, et al. Myelopreservation with the CDK4/6 inhibitor trilaciclib in patients with small-cell lung cancer receiving first-line chemotherapy: a phase Ib/randomized phase II trial. Ann Oncol 2019;30:1613-21.

30. Hart LL, Andric ZG, Hussein MA, et al. Effect of trilaciclib, a CDK 4/6 inhibitor, on myelosuppression in patients with previously treated extensive-stage small cell lung cancer receiving topotecan. J Clin Oncol 2019;37:abstr 8505.

31. Deng J, Wang ES, Jenkins RW, et al. CDK4/6 Inhibition Augments Antitumor Immunity by Enhancing T-cell Activation. Cancer Discov 2018;8:216-33.

32. Ojima I, Lichtenthal B, Lee S, et al. Taxane anticancer agents: a patent perspective. Expert Opin Ther Pat 2016;26:1-20.

33. Gelsomino F, Tiseo M, Barbieri F, et al. Phase II study of NAB-paclitaxel in sensitive and refractory relapsed SCLC (NABSTER TRIAL). Ann Oncol 2018;29:viii599.

34. Kang MH, Wang J, Makena MR, et al. Activity of MM398, nanoliposomal irinotecan (nal-IRI), in Ewing's family tumor xenografts is associated with high exposure of tumor to drug and high SLFN11 expression. Clin Cancer Res 2015;21:1139-50.

35. Paz-Ares LG, Spigel DR, Zielinski C, et al. RESILIENT: Study of irinotecan liposome injection (nal-IRI) in patients with small cell lung cancer-Preliminary findings from part 1 dose-defining phase. J Clin Oncol 2019;37:8562.

36. Genestreti G, Tiseo M, Kenmotsu H, et al. Outcomes of Platinum-Sensitive Small-Cell Lung Cancer Patients Treated With Platinum/Etoposide Rechallenge: A MultiInstitutional Retrospective Analysis. Clin Lung Cancer 2015;16:e223-8.

37. Morgensztern D, Rose M, Waqar SN, et al. RRx-001 followed by platinum plus etoposide in patients with previously treated small-cell lung cancer. Br J Cancer 2019;121:211-7.

38. Rudin CM, Pietanza MC, Bauer TM, et al. Rovalpituzumab tesirine, a DLL3-targeted antibody-drug conjugate, in recurrent small-cell lung cancer: a first-inhuman, first-in-class, open-label, phase 1 study. Lancet Oncol 2017;18:42-51.

39. Morgensztern D, Besse B, Greillier L, et al. Efficacy and Safety of Rovalpituzumab Tesirine in ThirdLine and Beyond Patients with DLL3-Expressing, Relapsed/Refractory Small-Cell Lung Cancer: Results From the Phase II TRINITY Study. Clin Cancer Res 2019;25:6958-66.

40. Komarnitsky PB, Lee H-J, Shah M, et al. A phase III study of rovalpituzumab tesirine maintenance therapy following first-line platinum-based chemotherapy in patients with extensive disease small cell lung cancer (ED SCLC). J Clin Oncol 2017;35:abstr TPS8583.

41. Smit MAD, Borghaei H, Owonikoko TK, et al. Phase 1 study of AMG 757, a half-life extended bispecific T cell engager (BiTE) antibody construct targeting DLL3, in patients with small cell lung cancer (SCLC). J Clin Oncol 2017;35:abstr TPS8577.

42. Giffin MJ, Lobenhofer EK, Cooke K, et al. Abstract 3632: BiTE® antibody constructs for the treatment of SCLC. Cancer Res 2017;77:abstr 3632.

43. Giffin M, Cooke K, Lobenhofer E, et al. P3.12-03 
Targeting DLL3 with AMG 757, a BiTE® Antibody Construct, and AMG 119, a CAR-T, for the Treatment of SCLC. J Thorac Oncol 2018;13:S971.

44. Byers LA, Chiappori A, Smit MAD. Phase 1 study of AMG 119, a chimeric antigen receptor (CAR) $\mathrm{T}$ cell therapy targeting DLL3, in patients with relapsed/refractory small cell lung cancer (SCLC). J Clin Oncol 2017;35:abstr TPS8576.

45. Russo A, Ron DA, Rasschaert M, et al. Is There Room for Personalized Medicine in Small-Cell Lung Cancer (SCLC)? Remarkable Activity of Pazopanib in Refractory FGFR1-Amplified ED-SCLC. JCO Precis Oncol 2019;3:1-8.

46. Salven P, Ruotsalainen T, Mattson K, et al. High pretreatment serum level of vascular endothelial growth factor (VEGF) is associated with poor outcome in small-cell lung cancer. Int J Cancer 1998;79:144-6.

47. Tiseo M, Boni L, Ambrosio F, et al. Italian, Multicenter, Phase III, Randomized Study of Cisplatin Plus Etoposide

Cite this article as: Serzan MT, Farid S, Liu SV. Drugs in development for small cell lung cancer. J Thorac Dis 2020;12(10):6298-6307. doi: 10.21037/jtd-2019-sclc-10
With or Without Bevacizumab as First-Line Treatment in Extensive-Disease Small-Cell Lung Cancer: The GOIRCAIFA FARM6PMFJM Trial. J Clin Oncol 2017;35:1281-7.

48. Ready NE, Pang HH, Gu L, et al. Chemotherapy With or Without Maintenance Sunitinib for Untreated ExtensiveStage Small-Cell Lung Cancer: A Randomized, DoubleBlind, Placebo-Controlled Phase II Study-CALGB 30504 (Alliance). J Clin Oncol 2015;33:1660-5.

49. Han B, Li K, Zhao Y, et al. Anlotinib as a third-line therapy in patients with refractory advanced non-smallcell lung cancer: a multicentre, randomised phase II trial (ALTER0302). Br J Cancer 2018;118:654-61.

50. Cheng Y, Wang Q, Li K, et al. Overall survival (OS) update in ALTER 1202: Anlotinib as third-line or furtherline treatment in relapsed small-cell lung cancer (SCLC). Ann Oncol 2019;30:v711.

51. Rudin CM, Poirier JT, Byers LA, et al. Molecular subtypes of small cell lung cancer: a synthesis of human and mouse model data. Nat Rev Cancer 2019;19:289-97. 